\title{
SIMPLICIAL GEOMETRY AND TRANSPORTATION POLYTOPES
}

\author{
BY
}

ETHAN D. BOLKER

\begin{abstract}
The classical transportation problem is the study of the set of nonnegative matrices with prescribed nonnegative row and column sums. It is aesthetically satisfying and perhaps potentially useful to study more general higher dimensional rectangular arrays whose sums on some subarrays are specified. We show how such problems can be rewritten as problems in homology theory. That translation explains the appearance of bipartite graphs in the study of the classical transportation problem. In our generalization, higher dimensional cell complexes occur. That is why the general problem requires a substantial independent investigation of simplicial geometry, the name given to the class of theorems on the geometry of a cell complex which depend on a particular cellular decomposition. The topological invariants of the complex are means, not ends. Thus simplicial geometry attempts to do for complexes what graph theory does for graphs. The dual title of this paper indicates that we shall spend as much time studying simplicial geometry for its own sake as applying the results to transportation problems. Our results include formulas for inverting the boundary operator of an acyclic cell complex, and some information on the number of such subcomplexes of a given complex.
\end{abstract}

1. Introduction. We begin our study with a careful statement of the general transportation problem. Notation has been chosen to simplify the reformulation of the problem as one in homology theory in §2. This paper is self-contained, although many of the graph theoretical precursors of the simplicial geometric techniques introduced here appear, in different notation, in [1] $\left({ }^{1}\right)$

Fix integers $r$ and $n_{0}, n_{1}, \ldots, n_{r}$. Let $N_{0}, N_{1}, \ldots, N_{r}$ be disjoint sets with cardinalities $\# N_{i}=n_{i}$. Then an $n_{0} \times \cdots \times n_{r}$ "matrix" is just a function $f$ on the cartesian product $K=N_{0} \times \cdots \times N_{r}$. Let $\Delta=\{0,1, \ldots, r\}$. Then

Received by the editors November 18, 1974.

AMS (MOS) subject classifications (1970). Primary 05B35, 05C05, 55J99; Secondary 05C30, $90 \mathrm{~B} 10$.

Key words and phrases. Simplicial geometry, transportation polytope, combinatorial geometry, matroid, tree, homology of finite complex.

(1)This seems a reasonable place to correct three errors in [1]. Equation (1) should read " $x_{11}=r_{1}+c_{1}, x_{1 j}=c_{j}(j>1), x_{i 1}=r_{i}(i>1), x_{i j}=0$ otherwise." In the last line on p. 253,18 should be 12 . At the bottom of p. $261, p(q ; 6,3)$ should be $64 q^{2}+$ $954 q+180$ rather than $64 q^{2}+834 q+120$. This last error was discovered by Dean Bandes, whose work on counting vertices in the classical problem will appear elsewhere. 
for any subset $I \subset \Delta$ there is a natural projection

$$
\pi_{I}: K \rightarrow \prod_{i \in I} N_{i}=\pi_{I}(K)
$$

A $\bar{j}$-subarray is a pair $(I, \tau)$ with $\tau \in \pi_{I}(K)$ for some $I$ for which $\# I=r+1-j$. We often write $\tau$ for $(I, \tau)$. Let

$$
K_{r-j}=\bigcup\left\{\pi_{I}(K) \mid \# I=r+1-j\right\}
$$

be the set of $j$-subarrays. Let $P_{i}=P_{i}\left(n_{0}, \ldots, n_{r}\right)$ be the elementary symmetric function of degree $i$ in the $r+1$ variables $n_{0}, \ldots, n_{r}$. Then there are $P_{r+1-j}$ $j$-subarrays. A $j$-sum transportation problem is a nonnegative function $g$ on $K_{r-j}$. The problem is to describe the answer, the set $M(g)$ of nonnegative matrices $f$ for which for all $\tau \in K_{r-j}$,

$$
\sum_{\sigma \in K ; \pi_{I}(\sigma)=\tau} f(\sigma)=g(\tau)
$$

The left member of (2) is called a $j$-sum of $f$. When $j$ is 1 or 2 we naturally speak of line or plane sums.

There are necessary conditions $g$ must satisfy in order that there be any function $f$ whose $j$-sums are given by $g$. We shall see what they are, and that they suffice, in $\S \S 2$ and 5 . Then $M(g)$ is a bounded convex polytope in $\mathbf{R}^{K}$. Describing $M(g)$ is nontrivial even in the classical case $r=j=1$ (see [1] and [5]). What we shall do is to find a topological setting in which the vertices of $M(g)$ correspond to some acyclic subcomplexes of a cell complex. Then we will be able to compute the dimension of $M(g)$ when it is not empty. Partial information about when it cannot be empty is obtained as well. Then for the cases in which no more than two of the $n_{i}$ exceed 2 , we will show that the most symmetrical line sum problems have answers with the maximum number of vertices, and find an upper bound for that number by a "tree count" for simplicial complexes analogous to Cayley's tree count for graphs. When more than two $n_{i}$ exceed 2 , topological questions of torsion and shellability enter in an essential way which is not yet understood. Better understanding might yield more information on orthogonal latin squares [4]. But before we turn to topology, we shall sketch a hypothetical problem in which line sums are specified.

Let $N_{0}, N_{1}$ and $N_{2}$ be sets of sources, sinks and commodities respectively. Let $g$ be a positive function on 1-subarrays (lines) which we interpret as follows:

$$
\begin{aligned}
g\left(v_{0}, v_{1}\right)= & \text { capacity of channel from source } v_{0} \in N_{0} \\
& \text { to sink } v_{1} \in N_{1} . \\
g\left(v_{0}, v_{2}\right)= & \text { quantity of commodity } v_{2} \text { available } \\
& \text { at source } v_{0} .
\end{aligned}
$$




$$
\begin{aligned}
g\left(v_{1}, v_{2}\right)= & \text { quantity of commodity } v_{2} \text { to be } \\
& \text { consumed at sink } v_{1} .
\end{aligned}
$$

A nonnegative $f$ with line sums $g$ is a feasible scheme: move $f\left(v_{0}, v_{1}, v_{2}\right)$ units of commodity $v_{2}$ to sink $v_{1}$ from source $v_{0}$. If the cost of a scheme is a concave function then the cheapest scheme is one of the vertices of $M(g)$. In order that there be any schemes, nonnegative (feasible) or not, $g$ must satisfy three kinds of conditions. One kind is that

$$
\sum_{v_{1} \in N_{1}} g\left(v_{0}, v_{1}\right)=\sum_{v_{2} \in N_{2}} g\left(v_{0}, v_{2}\right)
$$

for each fixed source $v_{0}$. That says that the total capacity of the channels leaving each source is just what is required to move all the commodities available there. We leave it to the reader to formulate the other two kinds of conditions. When those conditions are also satisfied there will always be a matrix $f$ whose line sums are given by $g$, but there need not always be a nonnegative $f$. Schemes with negative values can be thought of as requiring rerouting. We shall irivestigate when $M(g)$ can be empty at the end of $\S 3$.

It is not clear whether the theorems which follow are of practical significance in finding feasible schemes for problems like this. There are no algorithms presented. Perhaps this geometric rather than computational view of transportation problems will help others devise algorithms. Our job now is to rewrite the line sum problem as a problem in homology theory.

2. Multipartite complexes. Let $K\left(N_{0}, \ldots, N_{r}\right)$ be the $r$-dimensional simplicial complex which is the join of the disjoint vertex sets $N_{0}, \ldots, N_{r}$. That is, the $r$-simplexes of $K\left(N_{0}, \ldots, N_{r}\right)$ are the simplexes with one vertex in each $N_{i}$. They are thus in one to one correspondence with $K=N_{0} \times \cdots \times N_{r}$. We shall identify $K\left(N_{0}, \ldots, N_{r}\right)$ with $K$, the set of its $r$-simplexes. For $d \leqslant r$, the $d$-simplexes of $K$ are the faces of its $r$-simplexes. They are just the elements of the partial products $\pi_{I}(K)=\Pi_{i \in I} N_{i}$. Thus $K_{d}$, which we defined in the introduction as the set of $r-d$-subarrays, is the $d$-skeleton of $K$ when $K$ is regarded as a simplicial complex. Here our usage varies slightly from topological tradition, where the $d$-skeleton would denote the complex of all simplexes of $K$ of dimension not exceeding $d$. We augment $K$ by allowing a unique $(-1)$-simplex. When $r=1, K$ is the complete bipartite graph on the vertex sets $N_{0}, N_{1}$. When $r=$ $n_{0}=n_{1}=n_{2}=2, K$ is an octahedron.

Lemma 1. The $d$-simplex $(I, \tau)$ is a face of $\sigma \in K$ just when $\pi_{I}(\sigma)=\tau$, or, equivalently, when $\sigma$ lies on the subarray $\tau$.

Proof. Just unravel the definitions. 
Each $r$-simplex of $K$ comes equipped with an orientation since its vertices form an ordered $r+1$-tuple. We call this orientation of $K$ coherent because every $r-1$-simplex of $K$ inherits the same orientation from each $r$-simplex of which it is a facet. (A facet is a face of codimension 1.) To see that, suppose $\tau \in K_{r-1}$ is an $r-1$-simplex with no vertex in $N_{i}$. Then $\tau$ inherits the orientation $(-1)^{i}$ from each of the $n_{i}$ simplexes $\sigma$ with $\pi_{I}(\sigma)=\tau$. Thus the facets of the octahedron above are oriented so that no edges cancel.

A simplicial complex has a coherent orientation if and only if it is vertex $(r+1)$-colorable so that every $r$-simplex has one vertex of each color. Consequently, as Andrew Gleason observed, the barycentric subdivision of any simplicial complex has a coherent orientation, and can be regarded as a subcomplex of $K\left(N_{0}, \ldots, N_{r}\right)$ for suitable sets $N_{i}$. (For graphs, that statement says merely that if you insert a new vertex in the center of each edge of a graph the resulting graph is 2-colorable, hence bipartite.) Therefore the existence of a coherent orientation for a complex has no topologically invariant geometric significance. However, for us the particular triangulation matters. We are about to see that a coherently orientable triangulation allows us to compute $(r-1)$-boundaries as ordinary incidence sums, ignoring signs.

We write $C_{d}(K)$ for the $d$-chains on $K$. They are just functions on $K_{d}$, but we shall follow topological usage and write $f \in C_{d}(K)$ as a formal sum

$$
f=\sum_{\sigma \in K_{d}} f(\sigma) \cdot \sigma .
$$

The $r$-chains on $K$ are just the matrices we studied in $\$ 1$. The next easy theorem explains the point of what seems a digression into topology.

THEOREM 2. When the matrix $f$ is regarded as an r-chain on $K$ then its line sums are just the coefficients of the $r-1$-chain $\partial f$.

Proof. In the sum $\partial f=\Sigma_{\sigma \in K} f(\sigma) \partial \sigma$ collect the terms corresponding to each $\tau \in K_{r-1}$. Since $K$ is coherently oriented, the result is

$$
\partial f(\tau)=\sum\{f(\sigma) \mid \tau \text { a facet of } \sigma\} .
$$

The theorem follows when we use Lemma 1 to couple equations (2) and (3).

We shall study $j$-sums for $j>1$ in $\S 5$. We know now that the line sum problem is equivalent to specifying a $g \in C_{r-1}(K)$ and asking about $\partial^{-1} g \subset C_{r}(K)$. That is a question about the homology of $K$. In order for $\partial^{-1} g$ to be nonempty it is necessary that $\partial g=0$ in $C_{r-2}(K)$. That is, $g$ must be an $r-1$-cycle, an element of $Z_{r-1}(K)$. Those are the necessary conditions we mentioned in the introduction. They generalize equations (2.15) and (2.16) in [4]. When $r=1, g \in Z_{0}(K)$ just when the sum of the specified row sums equals the sum of 
the specified column sums. When $r \geqslant 2$ the condition $\partial g=0$ asserts the same thing for each 2-subarray.

To discover whether the necessary condition suffices we must compute $H_{r_{-1}}(K)$. The next lemma and theorem may seem obvious to a topologist, but they were not to me, a functional analyst turned combinatorist, so I include proofs. They illuminate the geometry of $K$ while computing its homology.

LEMMA 3. Let $K$ be a cell complex and $N$ a finite set disjoint from $K$. Let $K * N$ be the join of $K$ and $N$. Then

$$
H_{d}(K * N) \approx\left(H_{d-1}(K)\right)^{\# N-1} .
$$

Proof. Suppose $\# N=1$, so $N=\{v\}$. Then $K_{0}=K * N$ is the cone over $K$. It is contractible, hence for all $d, H_{d}\left(K_{0}\right)=\{0\}$. Note that even $H_{0}\left(K_{0}\right)=\{0\}$ since $K$ is augmented.

Suppose $\# N=2$; say $N=\{v, w\}$. Then $K_{1}=K * N$ is the suspension of $K$ and the theorem is true.

Finally, when $\# N>2$ we proceed by induction on $\# N$. Let $N=\{v, w, \ldots\}$, define $K_{0}$ and $K_{1}$ as above and let $K_{2}=K *(N-\{w\})$, for which we may assume the theorem is true. Then $K_{1} \cup K_{2}=K * N$ and $K_{1} \cap K_{2}=K_{0}$ so the Mayer-Vietoris sequence

$$
\cdots \rightarrow H_{d}\left(K_{0}\right) \rightarrow H_{d}\left(K_{1}\right) \times H_{d}\left(K_{2}\right) \rightarrow H_{d}(K * N) \rightarrow H_{d-1}\left(K_{0}\right) \rightarrow \cdots
$$

is exact. Since all homology of $K_{0}$ vanishes we have, in all dimensions $d$,

$$
H_{d}(K * N) \approx H_{d}\left(K_{1}\right) \times H_{d}\left(K_{2}\right)
$$

and the theorem follows.

The multipartite complexes $K$ we are interested in are just such multiple suspensions.

THEOREM 4. $H_{d}(K)$ is free of rank $\left(n_{0}-1\right) \cdots\left(n_{r}-1\right)$ if $d=r$ and is $\{0\}$ otherwise.

Proof. The theorem is true when $r=0$ because for an augmented complex, $H_{0}$ is free of rank one less than the number of components. Lemma 3 applied $r-1$ times then proves the theorem.

The Euler characteristic $\chi$ of $K$ is the alternating sum both of the ranks of $H_{d}(K)$ and of the numbers of $d$-simplexes, so .

$$
\chi=\left(n_{0}-1\right) \cdots\left(n_{r}-1\right)=P_{r+1}-P_{r}+P_{r-1}-\cdots .
$$

The last equality is obvious when we expand the product. The whole computation of $\chi$ is then an immediate consequence of the fact that all the homology groups of $K$ except $H_{r}(K)$ are $\{0\}$. 
Now we wish to study the affine geometry of the chain groups of $K$. For us, $C_{r}(K, \mathbf{R})$ and $C_{r-1}(K, \mathbf{R})$ are not abstract vector spaces. Our fixed, coherent orientation gives us particular bases in those spaces. We are interested in how the affine flat $\partial^{-1} g$ meets the positive orthant in $C_{r}(K, \mathrm{R})$ : the intersection is the answer $M(g)$ to the line sum problem $g$.

COROllaRY 6. For every $g \in Z_{r-1}(K, \mathbf{R}), \partial^{-1} g$ is a flat in $C_{r}(K)$ of dimension $\chi$.

PROOF. Since $H_{r-1}(K)=\{0\}$, every $r-1$-cycle is a boundary. Since there are no boundaries in dimension $r, H_{r}(K) \approx Z_{r}(K) \subset C_{r}(K)$ is just the kernel of $\partial$; it has dimension $\chi \cdot \partial^{-1} g$ is just a translate of that kernel.

Now to study $M(g)$ we study how the coordinate hyperplanes in $C_{r}(K, \mathbf{R})$ intersect in $\partial^{-1} g$. For each $\sigma \in K$ let

$$
V_{\sigma}=V_{\sigma}(g)=\left\{f \in \partial^{-1} g \mid f(\sigma)=0\right\} .
$$

Then $\left\{V_{\sigma}\right\}$ is an arrangement of $n_{0} \cdots n_{r}$ hyperplanes in the flat $\partial^{-1} g$, which has dimension $\chi=\left(n_{0}-1\right) \cdots\left(n_{r}-1\right)$. We shall study that arrangement.

3. Simplicial geometry: Inverting $\partial$. In this section we fashion tools for inverting the boundary operator of a cell complex. The constructions generalize those used to solve the classical transportation problem in [1]. I found clues to the generalizations in the study of simplicial geometry begun by Crapo and Rota [3]. We shall study here cell complexes more general than the multipartite simplicial complexes of $\S 2$ for two reasons. First, we will want to apply the results to study $j$-sum problems in $\S 5$. Second, the material is intrinsically interesting. It tells how to invert $\partial$ when $\partial$ is injective, and should be useful for more than the study of transportation problems. We shall try to follow the language of [6] for cell complexes.

Let $K$ be a finite, normal, regular, augmented cell complex which is purely $r$-dimensional: every $d$-cell for $d<r$ is a face of some $r$-cell. As usual, we shall identify $K$ with the set of its $r$-cells. Whenever $\sigma, \sigma^{\prime} \in K, \sigma \cap \sigma^{\prime}$ is a face of $\sigma$. We write $K_{d}$ for the set of $d$-cells of $K$ and call $K_{d}$ the $d$-skeleton. Suppose $K$ is coherently oriented, so that every $\tau \in K_{r-1}$ inherits the same orientation from each $\sigma \in K$ of which it is a facet. The multipartite complexes of $\S 2$ are examples of such cell complexes in which each $d$-cell is a $d$-simplex.

For each cell complex $K$ the chain complex $C_{r}(K) \stackrel{\partial}{\longrightarrow} C_{r-1}(K) \stackrel{\partial}{\longrightarrow} \cdots$ is well defined; in it the spaces of $r$ - and $r-1$-chains have natural bases, so we may speak of nonnegative chains. Moreover, (3) remains true: for $f \in C_{r}(K)$ and $\tau \in K_{r-1}$,

$$
\partial f(\tau)=\sum\{f(\sigma) \mid \tau \text { a facet of } \sigma\}
$$


Much of what follows can be proved for chain complexes with prescribed bases for $C_{r}$ and $C_{r-1}$. But that much generality is not useful now and replacing geometry by algebra would obscure much of the motivation.

Whenever $L \subset K$ we shall identify $L$ with the subcomplex of $K$ whose $r$-cells are the elements of $L$ and whose $d$-cells, for $d<r$, are all the $d$-cells of $K$. In particular, $K$ and $L$ have the same $r-1$-skeleton. When $d<r, C_{d}(L)=C_{d}(K)$, while $C_{r}(L)$ is the subspace of $C_{r}(K)$ containing those $r$-chains supported by $L$. We write $\beta_{d}(L)=\operatorname{rank} H_{d}(L)$ and $\chi(L)=\Sigma_{i}(-1)^{i} \beta_{r-i}(L)$ for the Betti numbers and Euler characteristic of $L$. For $\chi(K)$ we write just $\chi$. We shall also assume $\beta_{d}(K)=0$ for $d<r$. That makes the formulas which follow cleaner. It is easy to restore their more general form when necessary.

THEOREM 7. For subcomplexes $L \subset K$,

$$
\beta_{r}(L)-\beta_{r-1}(L)=\chi-\#(K-L)=\chi(L) .
$$

Proof. Since $L_{r-1}=K_{r-1}, H_{d}(L)=H_{d}(K)=\{0\}$ and thus $\beta_{d}(L)=0$ for $d \leqslant r-2$. Thus the left member of $(7)$ is $\chi(L)$. But $\chi(L)$ is also the alternating sum

$$
\begin{aligned}
\chi(L) & =\# L+\sum_{i \geq 1}(-1)^{i} \#\left(L_{r-i}\right) \\
& =\# K-\#(K-L)+\sum_{i \geq 1}(-1)^{i} \#\left(K_{r-i}\right) \\
& =\chi-\#(K-L) . \quad \square
\end{aligned}
$$

Think of Theorem 7 as saying "When you remove the interior of an $r$-cell of $K$ you either kill an $r$-cycle, decreasing $\beta_{r}$ by 1 , or you prevent an $r-1$-cycle which used to bound from so doing, increasing $\beta_{r-1}$ by 1 ."

When $\beta_{r}(L)=0, L$ is independent; when $\beta_{r-1}(L)=0, L$ spans. That usage is suggested by combinatorial geometry. When $r=1, L$ is a graph. It is then independent when it is free of loops, and spans when it is connected. A subcomplex $T$ which is independent and spans is, of course, a basis. Bases are almost acyclic: all the Betti numbers of a basis are 0 , but a basis may have torsion. When $r=1$, so that $K$ is a graph, a basis is known as a tree. That is the nomenclature we shall use for $r>1$ as well. It follows from Theorem 7 that all trees in $K$ are equicardinal: since $\chi(T)=0, \chi=\#(K-T)$ and thus $\# T=$ $\# K-\chi$. For multipartite complexes trees contain

$$
n_{0} \cdots n_{r}-\left(n_{0}-1\right) \cdots\left(n_{r}-1\right)=\sum_{i \geqslant 0}(-1)^{i} P_{r-i}
$$

simplexes.

Write $\partial_{L}$ for the boundary operator of the subcomplex $L$. If $g \in Z_{r_{-1}}(K)$ 
$=Z_{r-1}(L)$ then $\partial_{L}^{-1}(g)$ is the set of $r$-cycles $f$ of $K$ with support $L$ and $\partial f=g$. In the language of $\S 2, \partial_{L}^{-1}(g)=\bigcap_{\sigma \notin L} V_{\sigma}$.

Corollary 8. When $\beta_{r-1}(L)=0$,

$$
\operatorname{dim} \partial_{L}^{-1}(g)=\chi-\#(K-L)=\beta_{r}(L) .
$$

Proof. This is Theorem 6 of [1], which wàs left unproved there. It is now obvious.

CoRollary 9. When $T$ is a tree in $K, \partial_{T}^{-1}(g)$ is a vertex of the arrangement $\left\{V_{\sigma}\right\}$ of hyperplanes in $\partial^{-1}$.

Proof. Corollary 8 shows $\partial_{T}^{-1}(g)$ has dimension zero.

Let $T$ be a tree in $K$. Then for $g \in Z_{r-1}$ there is a unique $f \in C_{r}(T)$ with $\partial f=g$. We want formulas expressing the numbers $f(\sigma)$ in terms of $g$. To that end we study maximal nonspanning subcomplexes. We say $A \subset K$ is a copoint if $\beta_{r-1}(A)=1$ and $A$ is maximal with that property. When $K=K(M, N)$ is a complete bipartite graph, a copoint is a disjoint union of two complete bipartite subgraphs. It is determined by partitions $\left\{A, A^{\prime}\right\}$ and $\left\{B, B^{\prime}\right\}$ of $M$ and $N$ respectively. The name "copoint" is from combinatorial geometry. I do not know whether topologists have made sufficient use of the notion to name it.

Let $A$ be a copoint. Then $H_{r-1}(A, \mathrm{Z}) \approx \mathrm{Z} \times G$, where $G$ is a finite group. Choose a generator $\zeta$ of the free part of $H_{r-1}$. Let $\sigma$ be an $r$-cell of $K$. Then $\partial \sigma \in Z_{r-1}(A)$, so there is an integer $m(\sigma, A)$ and a $\gamma \in G$ such that

$$
\partial \sigma \sim(m(\sigma, A) \zeta, \gamma)
$$

Here " $\sim$ " means "homologous in $H_{r-1}(A, \mathrm{Z})$." The nonnegative integer $|m(\sigma, A)|$ is the multiplicity of $\sigma$ at $A$. Only $|m(\sigma, A)|$ is well defined, for the opposite choice of generator reverses the sign of $m(\sigma, A)$.

LEMma 10. The integer $|m(\sigma, A)|$ is the order of $\zeta$ in $H_{r-1}(A \cup\{\sigma\})$. $m(\sigma, A)=0$ if and only if $\sigma \in A$.

Proof. We have

$$
H_{r-1}(A \cup\{\sigma\}) \approx \mathrm{Z} /(m(\sigma, A)) \times G /(\gamma) .
$$

That group has rank 1 (respectively 0 ) when $\sigma \in A$ (respectively $\sigma \notin A$ ).

Now suppose $g \in Z_{r-1}(K, \mathbf{R})$. Then in $H_{r-1}(A, \mathbf{R}) \approx \mathbf{R}, g$ is homologous to a scalar multiple of $\zeta$. That multiple is unique and depends linearly on $g$. Thus we have constructed a linear functional $\mu_{A}: Z_{r-1} \rightarrow \mathbf{R}$ such that

$$
g \sim \mu_{A}(g) \zeta \text { in } H_{r-1}(A, \mathbf{R}) .
$$

When $K$ is a bipartite graph the functionals $\mu_{A}$ are what I called $\mu_{A, B}$ in [1, Equation (4)]. 
THEOREM 11. Let $f \in C_{r}(K, \mathbf{R})$. Then

$$
\mu_{A}(\partial f)=\sum_{\sigma \in K} f(\sigma) m(\sigma, A)=\sum_{\sigma \notin A} f(\sigma) m(\sigma, A) .
$$

Proof. We have

$$
\partial f=\sum_{\sigma \in K} f(\sigma) \partial \sigma \sim \sum_{\sigma \in K} f(\sigma) m(\sigma, A) \zeta ;
$$

the first half of the theorem then follows from the definition of $\mu_{A}$. The second half is a consequence of Lemma 10.

Corollary 12. $m(\sigma, A)=\mu_{A}(\partial \sigma)$.

Proof. Apply Theorem 11 to the $f \in C_{r}$ which is 1 at $\sigma$ and 0 otherwise.

CoRollary 13. Suppose $\sigma \notin A, f \in C_{r}(A \cup\{\sigma\})$ and $\partial f=g$. Then

$$
f(\sigma)=\mu_{A}(g) / m(\sigma, A) .
$$

Proof. Substitute in (12).

Note that the sign in (13) is correct and does not depend on the choice of generator $\zeta$, which affects the signs of the numerator and denominator the same way.

The copoint functionals tell us how to compute on trees.

THEOREM 14. Let $T$ be a tree and $\sigma \in T$. Then there is a unique copoint A containing $T-\{\sigma\}$. Then

$$
\partial_{T}^{-1} g(\sigma)=\mu_{A}(g) / m(\sigma, A) .
$$

Proof. Since $T$ is a tree, Theorem 7 implies $\beta_{r}(T-\{\sigma\})=0$ and $\beta_{r-1}(T-\{\sigma\})=1$. Then let

$$
A=\left\{\sigma^{\prime} \in K \mid \beta_{r-1}\left(T-\{\sigma\} \cup\left\{\sigma^{\prime}\right\}\right)=1\right\}=\operatorname{cl}(T-\{\sigma\}),
$$

the closure of $T-\{\sigma\}$ in the sense of combinatorial geometry. Then $T-\{\sigma\} \subset$ $A, \sigma \notin A$ and $T \subset A \cup\{\sigma\}$, so Corollary 13 applies to $\partial_{T}^{-1} g \in C_{r}(A \cup\{\sigma\})$.

When $T$ and $A$ are related as in Theorem 14 we say $T$ uses $A$. We have so far attached multiplicities to pairs $(\sigma, A)$, where $A$ is a copoint and $\sigma \notin A$. If $T$ is a tree we define the multiplicity of $\sigma$ in $T$ to be

$$
m(\sigma, T)=|m(\sigma, \mathrm{cl}(T-\{\sigma\}))| .
$$

A tree $T$ is multiplicity free when $m(\sigma, T)=1$ for all $\sigma \in T$.

LEMMA 15. A tree $T$ is multiplicity free if it is torsion free; that is, if $H_{r-1}(T)=\{0\}$

Proof. Suppose $\sigma \in T$. Let $A=\mathrm{cl}(T-\{\sigma\})$. Then $H_{r-1}(T-\{\sigma\})=$ 
$H_{r-1}(A)$ because adding to $T-\{\sigma\}$ those $r$-cells which increase $\beta_{r}$ does not change $H_{r-1}$. Then $H_{r-1}(T)=H_{r-1}(A \cup\{\sigma\})$. If that group is $\{0\}$ then (10) implies $|m(\sigma, A)|=1$.

We are ready to study the vertices of the answer

$$
M(g)=\left\{f \in C_{r}(K) \mid \partial f=g \text { and } f \geqslant 0\right\}
$$

to the problem $g \geqslant 0$. The tree $T$ is honest for the problem $g$ when the vertex $\partial_{T}^{-1} g$ of the arrangement $\left\{V_{\sigma}\right\}$ of hyperplanes is nonnegative.

THEOREM 16. The vertices of $M(g)$ are those vertices of $\left\{V_{\sigma}\right\}$ which correspond to honest trees.

Proof. Clearly every vertex of $M(g)$ corresponds to an honest tree. Conversely, the vertex corresponding to an honest tree is in $M(g)$ and must be one of its vertices.

For each copoint $A$ the kernel of $\mu_{A}$, which is well defined even though the sign of $\mu_{A}$ is ambiguous, is called the degeneracy hyperplane corresponding to $A$. The degeneracy hyperplanes carve $Z_{r-1}$ into open regions of nondegeneracy; $g$ is degenerate if for some $A, \mu_{A}(g)=0$.

THEOREM 17. As $g$ varies in $Z_{r-1}$, the combinatorial type of $M(g)$ is constant on regions of nondegeneracy.

Proof. On such a region none of the functions $\mu_{A}$ changes sign, and none is 0 . Hence the honest trees are the same throughout the region. The combinatorial type of a polytope is determined by the incidence relation between its vertices and its facets. The vertices of $M(g)$ we have just identified. The facets are the nonempty intersections $M(g) \cap V_{\sigma}$. Since $\partial_{T}^{-1} g \in V_{\sigma}$ if and only if $\sigma \notin$ $T$ the incidence relation and hence the combinatorial type is constant on regions of nondegeneracy.

We can study how honest trees become dishonest or vice versa as $g$ crosses a degeneracy hyperplane. Let us say two trees $T, T^{\prime}$ are in the same grove at a copoint $A$ when there are $r$-cells $\sigma, \sigma^{\prime}$ in $T, T^{\prime}$ respectively such that $T-\{\sigma\}=$ $T^{\prime}-\left\{\sigma^{\prime}\right\}$. If we start with a tree $T$ and a $\sigma \in T$ and let $A=\operatorname{cl}(T-\{\sigma\})$ then the other trees in the grove with $T$ at $A$ are the trees

$$
T^{\prime}=T-\{\sigma\} \cup\left\{\sigma^{\prime}\right\}, \quad \sigma^{\prime} \notin A .
$$

In the language of combinatorial geometry a simple basis exchange converts $T$ to $T^{\prime}$.

LEMMA 18. Let $R^{+}$and $R^{-}$be regions of nondegeneracy which share a facet lying on the degeneracy hyperplane $\operatorname{ker} \mu_{A}$. Adjust the sign of $\mu_{A}$ so that it is positive on $R^{+}$. Let $T$ be a tree honest on $R^{+}$. Then the tree $T^{\prime}=T-$ 
$\{\sigma\} \cup\left\{\sigma^{\prime}\right\}$ in the grove with $T$ at $A$ is honest on $R^{+}$or $R^{-}$according to whether $m\left(\sigma^{\prime}, A\right)>0$ or $m\left(\sigma^{\prime}, A\right)<0$.

Proof. Since $\partial_{T^{\prime}}^{-1} g(\sigma)=\mu_{A}(g) / m\left(\sigma^{\prime}, A\right)$ is positive for $g \in R^{+}$when $m\left(\sigma^{\prime}, A\right)>0$ and for $g \in R^{-}$when $m\left(\sigma^{\prime}, A\right)<0$ we need only show that $\partial_{T^{\prime}}^{-1} g$ is positive on $S=T-\{\sigma\}=T^{\prime}-\left\{\sigma^{\prime}\right\}$. Find a small ball $B$ which meets both the regions of nondegeneracy $R^{ \pm}$and no others. Then $B$ also meets ker $\mu_{A}$; we may suppose its center $g_{0}$ lies on ker $\mu_{A}$. Then $\partial_{T}^{-1} g$ is a continuous function of $g$ and is positive on $S$ throughout $B$. Thus on $S, \partial_{T^{\prime}}^{-1}\left(g_{0}\right)=\partial_{T}^{-1}\left(g_{0}\right)>0$. Since $\partial_{T^{\prime}}^{-1}$ is continuous too, it is positive on $S$ throughout $B$ or some smaller ball and hence is, in fact, positive on all of $R^{+} \cup R^{-}$.

Let $1 \in C_{r}(K)$ be the $r$-chain all of whose coefficients are 1: $1=\Sigma_{\sigma \in K} \sigma$. We call $\partial 1$ the center of $Z_{r-1}$; it is the most symmetrical positive problem. Let us choose the sign of each copoint functional $\mu_{A}$ so that $\mu_{A}(\partial 1) \geqslant 0$. Then Theorem 11 tells us

$$
\sum_{\sigma \notin A} m(\sigma, A)=\mu_{A}(\partial 1) \geqslant 0 .
$$

THEOREM 19. If $g_{0}$ is degenerate there is a nondegenerate problem $g$ near $g_{0}$ for which $M(g)$ has at least as many vertices as $M\left(g_{0}\right)$.

Proof. Suppose $\mu_{A}\left(g_{0}\right)=0$; for convenience we give the proof only for a $g_{0}$ which lies on just one degeneracy hyperplane. If a tree $T$ is honest at $g_{0}$ and does not use $A$ then it is honest for all $g$ near $g_{0}$. If a tree $T$ honest at $g_{0}$ uses $A$, then in the grove with $T$ at $A$ suppose there are $v$ trees $T \cap A \cup\{\sigma\}$ for which $m(\sigma, A)>0$. Equation (16) implies $v>0$. For $g$ near $g_{0}$ with $\mu_{A}(g)$ $>0$ there are thus $v$ distinct vertices of $M(g)$ which coalesce into the single vertex $\partial_{T}^{-1} g_{0}$ as $g$ approaches $g_{0}$.

THEOREM 20. Let $K$ be a cell complex in which all trees are multiplicity free. Let $R^{*}$ be a region of nondegeneracy whose closure contains the center d1. Then among all problems, those in $R^{*}$ have answers with the maximum number of vertices.

Proof. Let $g \in Z_{r-1}$ be any nondegenerate problem. Pick a problem $g^{*} \in R^{*}$ so that the directed line segment joining $g$ to $g^{*}$ crosses degeneracy hyperplanes one at a time. Suppose that at one such point we cross ker $\mu_{A}$ from $R^{-}$to $R^{+}$. Since $R^{+}$and $R^{*}$ lie on the same side of ker $\mu_{A}, \mu_{A}>0$ on $R^{+}$. Let $v^{+}\left(v^{-}\right)$be the number of vertices of the answer to any problem in $R^{+}\left(R^{-}\right)$. We shall show $v^{+}-v^{-} \geqslant 0$. Any tree $T$ which is honest on $R^{-}$and does not use $A$ is honest on $R^{+}$too and so does not contribute to the difference, so suppose $T$ uses $A$. Then the trees $T^{\prime}$ in the grove with $T$ at $A$ are given by (15). Lemma 18 shows that $T^{\prime}$ is honest on $R^{+}\left(R^{-}\right)$when $m\left(\sigma^{\prime}, A\right)=+1$ 
$(-1)$. Let $t$ be the number of groves at $A$ which contain a tree honest on $R^{+}$or $R^{-}$. Each of those groves consists entirely of such trees, and

$$
v^{+}-v^{-}=t\left(\sum_{\sigma^{\prime} \notin A} m\left(\sigma^{\prime}, A\right)\right)=t \mu_{A}(\partial 1) \geqslant 0 .
$$

When the center $\partial 1$ is nondegenerate, $M(\partial 1)$ has the maximum number of vertices. That is the case for $K\left(N_{0}, N_{1}\right)$ when $n_{0}$ and $n_{1}$ are relatively prime. For multipartite complexes with $r>1, \partial 1$ is degenerate if for some $i$ and $j$, g.c.d. $\left(n_{i}, n_{j}\right)>1$. I do not know if the converse is true.

The hypothesis in Theorem 20 that every tree be multiplicity free is quite restrictive. We shall see just how restrictive in the next section. I do not know whether the theorem is true without that hypothesis. There may be a way to salvage the proof by counting honest trees $T$ with multiplicities. The average or the maximum of the set of numbers $\{m(\sigma, T) \mid \sigma \in T\}$ suggest themselves but do not seem to work.

We close this section with an examination of a way in which $M(g)$ can be empty even when $g \in Z_{r-1}^{+}$, the set of nonnegative $r-1$-cycles.

LEMma 21. Let $\tau \in K_{r-1}$. Then the coordinate map $g \mapsto g(\tau)$ is a constant multiple of a copoint functional if and only if

$$
A_{\tau}=\{\sigma \in K \mid \tau \text { not a facet of } \sigma\}
$$

is a copoint.

Proof. Suppose $A_{\tau}$ is a copoint. Then for $\sigma, \sigma^{\prime} \notin A_{\tau}, \partial \sigma \sim \partial \sigma^{\prime}$ in $H_{r-1}\left(A_{\tau}, \mathbf{R}\right)$ since $\beta_{r-1}\left(A_{\tau}\right)=1$ and $\partial \sigma, \partial \sigma^{\prime}$ have the same coefficient, namely 1 , at $\tau$. Thus $m\left(\sigma, A_{\tau}\right)$ is a constant $c$ for $\sigma \notin A_{\tau}$. Then for all $\sigma$

$$
\begin{aligned}
\mu_{A_{\tau}}(\partial \sigma) & = \begin{cases}0, & \sigma \in A_{\tau}, \\
c, & \sigma \notin A_{\tau},\end{cases} \\
& =\operatorname{cg}(\partial \sigma) .
\end{aligned}
$$

Since the boundaries $\partial \sigma$ span $B_{r-1}=Z_{r-1},(17)$ implies $\mu_{A_{\tau}}(g)=c g(\tau)$ for all $g \in Z_{r-1}$.

Conversely, suppose there is a copoint $A$ and a constant $c$ such that $\mu_{A}(g)$ $=c g(\tau)$ for all $g \in Z_{r-1}$. Then for any $\sigma \in K$

$$
\begin{aligned}
m(\sigma, A) & =\mu_{A}(\partial \sigma)=c \partial \sigma(\tau) \\
& = \begin{cases}0 & \text { if } \sigma \in A_{\tau}, \\
c & \text { if } \sigma \notin A_{\tau} .\end{cases}
\end{aligned}
$$

But $m(\sigma, A) \neq 0$ just when $\sigma \notin A$, so $A=A_{\tau}$. 
COROLLARY 22. $Z_{r-1}^{+}$is a union of regions of nondegeneracy if and only if every $A_{\tau}$ is a copoint.

Proof. $Z_{r-1}^{+}$is such a union if and only if its bounding hyperplanes, the coordinate hyperplanes, are the kernels of copoint functionals.

COROLlarY 23. If for some $\tau, A_{\tau}$ is not a copoint, then there are problems $g \in Z_{r-1}^{+}$for which $M(g)$ is empty.

Proof. Choose a $g_{0} \in Z_{r-1}$ such that $g_{0}(\tau)=0, g_{0}\left(\tau^{\prime}\right)>0$ for $\tau^{\prime} \neq \tau$, and such that a neighborhood of $g_{0}$ lies in a region of nondegeneracy $R$. Then $R$ meets both $Z_{r-1}^{+}$and its complement. But when $g \notin Z_{r-1}^{+}$there can be no $f \geqslant 0$ with $\partial f=g$, so $M(g)$ is empty. Theorem 17 implies $M(g)$ is empty for all $g \in R$, and some of those $g$ lie in $Z_{r-1}^{+}$.

Unfortunately, Corollary 23 does not locate all the regions of nondegeneracy the answers to whose problems are empty. The following example is a perturbation of one in [4]. Consider the $2 \times 2 \times 2$ matrix given by

$$
f(\cdot, \cdot, 1)=\left(\begin{array}{rr}
7 & 3 \\
3 & -1
\end{array}\right), f(\cdot, \cdot, 2)=\left(\begin{array}{rr}
-1 & 3 \\
3 & 7
\end{array}\right) .
$$

It is easy to check that the line sums of $f$ are all positive. $K\left(N_{0}, N_{1}, N_{2}\right)$ is an octahedron when $n_{0}=n_{1}=n_{2}=2$. The trees $T$ are just the complexes $K-\{\sigma\}$ for each of the 8 triangles $\sigma$. Putting a 0 in each of those 8 places in turn and computing $\partial_{T}^{-1}(\partial f)$ shows $M(\partial f)$ has no vertices and hence is empty. To show that this case is not covered by Corollary 23, we prove Theorem 24 .

THEOREM 24. In the multipartite simplicial complex $K=K\left(N_{0}, \ldots, N_{r}\right)$, $n_{i} \geqslant 2$, every $A_{\tau}$ is a copoint, and $Z_{r-1}^{+}$is a union of regions of nondegeneracy.

Proof. Whenever $\sigma \notin A_{\tau}, \partial \sigma$ is not homologous to 0 in $A_{\tau}$ so $\beta=$ $\beta_{r-1}\left(A_{\tau}\right) \geqslant 1$. We need only show $\beta=1$. That is equivalent to showing that when $\sigma, \sigma^{\prime} \notin A_{\tau}, \sigma-\sigma^{\prime} \sim 0$ in $H_{r-1}\left(A_{\tau}, \mathbf{R}\right)$. Suppose $\tau \in K\left(N_{1}, \ldots, N_{r}\right)$, $\sigma=(v, \tau)$ and $\sigma^{\prime}=(w, \tau)$. Choose $\tau^{\prime} \in K\left(N_{1}, \ldots, N_{r}\right)$ sharing no vertices with $\tau$; that is possible since each $n_{i} \geqslant 2$. Let $C \subset K$ consist of all simplexes of $K$ whose vertices are among those of $\tau, \tau^{\prime}$, and $\{v, w\}$. Then $C$ is a cross polytope (the $r$-dimensional analogue of the octahedron, the join of $r$ two element sets), and $C \cap A_{\tau}=\left\{\sigma, \sigma^{\prime}\right\}$. In fact, $\sigma$ and $\sigma^{\prime}$ are adjacent facets of $C$, so when the facets of $C$ are oriented so as to make $C$ the boundary of an $r$-cell, $\sigma$ and $\sigma^{\prime}$ have opposite signs. Since $\partial C=0, \partial \sigma \sim \partial \sigma^{\prime}$ in $H_{r-1}\left(A_{\tau}\right)$.

We thus still lack a good criterion for deciding when $M(g)$ is empty. Techniques analogous to those developed in $[1, \S 1]$ may lead to one. I conjecture that if $M(g)$ is not empty it has dimension $\chi$, the maximum possible. In [4], 
Jurkat and Ryser show that when $r=2$ the answer to a positive plane sum problem is never empty. Their argument generalizes easily to prove the same for the answer to a positive $r$-sum problem.

4. Multipartite complexes with multiplicity free trees. In this section we prove that every tree of $K=K\left(N_{0}, \ldots, N_{r}\right)$ is multiplicity free if and only if at most two of the $n_{i}$ exceed 2, and count the number of trees in that case.

Construction 25. Suppose $L$ is a subcomplex of $K$. Let $N$ be a set of $n$ elements, disjoint from all the $N_{i}$. We shall systematically construct $n^{\chi-\chi(L)}$ subcomplexes $L^{*}$ of $K^{*}=K\left(N_{0}, \ldots, N_{r}, N\right)$ each of which satisfies

$$
H_{d}\left(L^{*}\right) \approx\left(H_{d-1}(L)\right)^{n-1}
$$

for all $d$.

To start the construction, let $L^{\prime}$ be the join $L * N . L^{\prime}$ is not yet a subcomplex of $K^{*}$ because its $r$-skeleton is too small; we shall repair that defect after we observe that Lemma 3 applies to $L^{\prime}$, so (18) is true for $L^{\prime}$ :

$$
H_{d}\left(L^{\prime}\right) \approx\left(H_{d-1}(L)\right)^{n-1} .
$$

How much of the $r$-skeleton of $K^{*}$ is missing from $L^{\prime}$ ? Suppose $\tau^{*} \in\left(K^{*}\right)_{r}$. If $\tau^{*} \notin K$ then $\tau^{*}$ is the join of a vertex of $N$ and an $r-1$-simplex $\tau$ of $K$. Then $\tau \in L_{r-1}=K_{r-1}$ so $\tau^{*} \in L_{r-1}^{\prime}$. If $\tau^{*}$ is one of the $\# L r$-simplexes of $L$ then it is a facet of $n r+1$-simplexes of $L^{\prime}$. But the other $\#(K-L)=\chi-\chi(L) r$-simplexes $\tau^{*}$ of $K$ are facets of no $r+1$-simplex of $L^{\prime}$. Each of these can be made such a facet by adjoining to $L^{\prime}$ any one of the $n r+1$-simplexes $\left(\tau^{*}, v\right)$, where $v \in N$. These adjunctions leave the homotopy type and hence the homology of $L^{\prime}$ unchanged. The $n^{\chi-\chi(L)}$ complexes $L^{*}$ which result from these independent choices are the ones whose existence we have asserted.

THEOREM 26. Suppose $n=2$. Then Construction 25 applied in turn to each of the trees of $K$ yields all the trees of $K^{*}$, once each. Whatever torsion appears in the trees of $K$ appears in the same proportion in those of $K^{*}$. If $K$ has $t$ trees, $K^{*}$ has $t \cdot 2^{\mathrm{X}(K)}$.

Proof. If $T$ is a tree of $K$ then $\chi(T)=0$ and each of the $2^{x(K)}$ subcomplexes $T^{*}$ of $K^{*}$ produced by Construction 25 is a tree for which $H_{r}\left(T^{*}\right)=$ $H_{r-1}(T)$, and the trees $T^{*}$ are clearly distinct. Conversely, let $T^{*}$ be a tree of $K^{*}$. Suppose $N=\{v, w\}$ and let $C_{v}$ be the star of $v$, the $r+1$-complex whose simplexes are all the simplexes of $T^{*}$ which have $v$ as a vertex. Note that $\left(C_{v}\right)_{r} \neq$ $K_{r}^{*}$. Similarly define $C_{w}$. Let $T=C_{v} \cap C_{w} \subset K$. We will be done once we have shown $T$ is a tree in $K$. Since $C_{v}$ and $C_{w}$ are cones, all their homology groups vanish. Then an easy application of the Mayer-Vietoris Theorem shows $H_{d}(T)=H_{d+1}\left(T^{*}\right)$ for all $d$. Thus $T$ has the right homology; we need only show 
$T_{r-1}=K_{r-1}$. Let $\tau$ be an $r-1$-simplex of $K$. Then $(\tau, v)$ and $(\tau, w)$ are $r$-simplexes of $K^{*}$ and thus each is a facet of some $r+1$-simplex in $T^{*}$. Hence $\tau \in$ $\left(C_{v} \cap C_{w}\right)_{r-1}$.

THEOREM 27. If $n_{0}=m, n_{1}=n$, and $n_{2}=n_{3}=\cdots=n_{r}=2$ then all the trees of $K$ are multiplicity free and there are

$$
m^{n-1} n^{m-1} 2^{(r-1) \times(K)}=m^{n-1} n^{m-1} 2^{(r-1)(m-1)(n-1)}
$$

trees.

Proof. When $r=1, K\left(N_{0}, N_{1}\right)$ is a bipartite graph. It is known to have $m^{n-1} n^{m-1}$ trees, all of which are torsion free and hence multiplicity free. Then Theorem 26 applied $r-1$ times and Lemma 15 imply Theorem 27.

THEOREM 28. If $n_{0}, n_{1}, n_{2} \geqslant 3$ and $n_{3}, \ldots, n_{r} \geqslant 2$, then $K$ contains $a$ tree $T$ and $a, \sigma \in T$ for which $m(\sigma, T)=2$.

Proof. Figure 1 shows the projective plane $P_{2}$ triangulated with 9 vertices so as to live in $K(X, Y, Z)$ where $\# X=\# Y=\# Z=3$. Then $K(X, Y, Z)$ is naturally a subset of $K\left(N_{0}, N_{1}, N_{2}\right)$. There we can extend $\mathrm{P}_{2}$ to a tree $T_{0}$ by adjoining enough triangles to fill out the 1-skeleton and reduce $\beta_{1}$ to 0 . Then $H_{1}\left(T_{0}\right) \approx \mathrm{Z}_{2}$. Apply Construction 25 repeatedly to $T_{0}$ to produce a tree $T$ in $K$ with $H_{r-1}(T) \approx Z_{2}^{p}, p=\left(n_{3}-1\right) \cdots\left(n_{r}-1\right)$. Choose a $g \in Z_{r-1}(T, Z)$ which is not homologous to 0 and an $f \in C_{r-1}(T)$ with minimal support for which $\partial f=2 g$. Then for any $\sigma$ in the support of $f, m(\sigma, T)=2$.

The presence of torsion, and hence of multiplicities, can cause $M(g)$ to have strange vertices. For example, consider $K(X, Y, Z)$ as in Theorem 28 and the line sum problem $g \equiv 1$. Then $M(g)$ is the analogue for $3 \times 3 \times 3$ matrices of the polytope of doubly stochastic $n \times n$ matrices. The vertices of that polytope have long been known to be the permutation matrices. Now let $T$ be the complex $\mathrm{P}_{2}$ shown in Figure 1 together with the triangles $X_{2} Y_{3} Z_{2}, X_{2} Y_{2} Z_{3}$ and $X_{2} Y_{2} Z_{2}$. Then $T$ is a tree. It is easy to check that $\partial_{T}^{-1}(g)$ is the unique 2-chain which has value $1 / 2$ on all the triangles in $P_{2}$, value 1 on $X_{2} Y_{2} Z_{2}$, and value 0 elsewhere. That 2-chain is a vertex of $M(g)$ which does not have integer entries. I suspect it is torsion and the resulting nonintegrality of vertices which helps make the search for orthogonal latin squares so hard [4].

It is tempting on numerological grounds to guess that, in general, $K$ has

$$
n_{0}^{x_{0}} n_{1}^{x_{1}} \cdots n_{r}^{x_{r}}
$$

trees, where

$$
\chi_{i}=\chi\left(K\left(N_{0}, \ldots, \hat{N}_{i}, \ldots, N_{r}\right)\right)=\prod_{j \neq i}\left(n_{j}-1\right)
$$




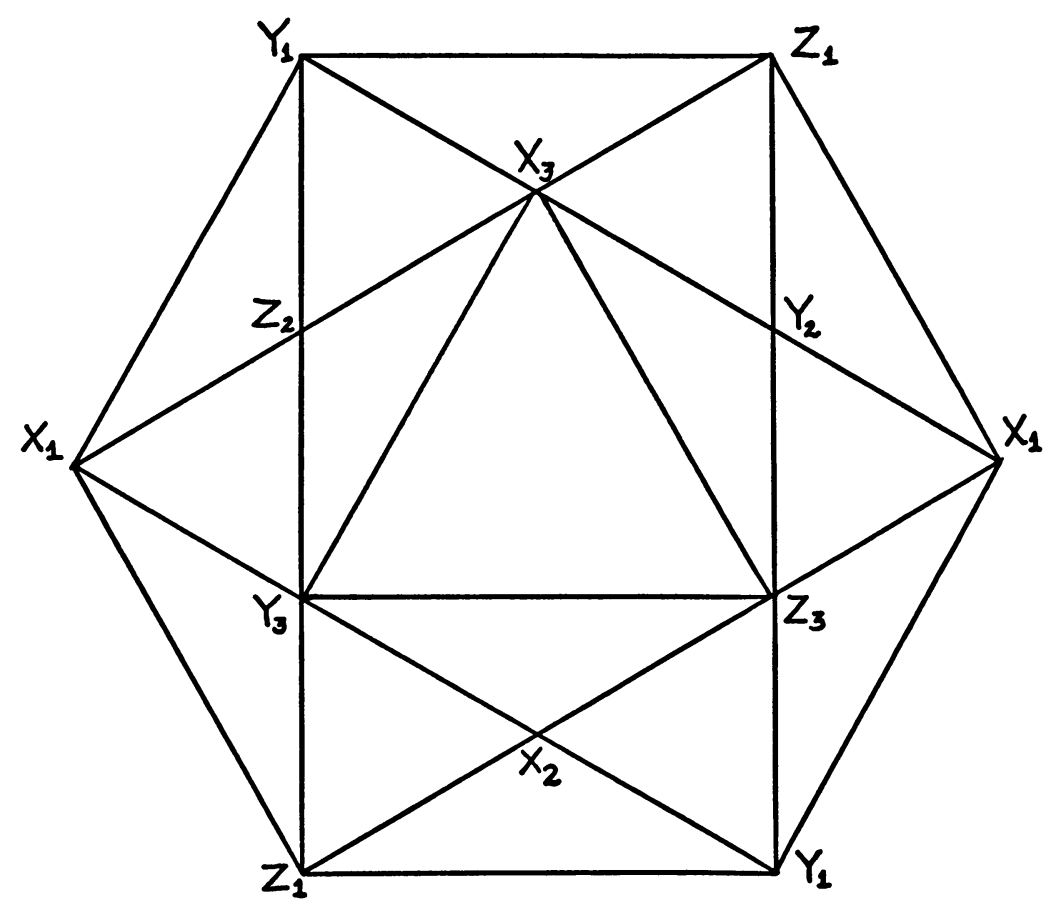

FIgURE 1

$\mathbf{P}_{2}$

A similarly tempting guess is that the $r$-skeleton of the $n-1$-simplex has

$$
n^{\left(\begin{array}{c}
n-2 \\
r
\end{array}\right)}
$$

trees. Both guesses are wrong. The second is right only when $n \leqslant 5$ or $r=0,1$, $n-2$ or $n-1$; the 6 point triangulation of $P_{2}$ fouls the tree count when $n=6$ and $r=2$. The first guess is right only when all the trees of $K$ are multiplicity free.

When trying to count trees, those with torsion do not present the only problem. Call a tree shellable if it can be built from a single r-simplex by successively attaching $r$-simplexes along contractible subcomplexes of their boundaries. When $r=1$, all trees can be shelled. Shellable trees are torsion free, but not conversely: the dunce's cap [8], exhibited in Figure 2 as a subcomplex of $K\left(N_{0}, N_{1}, N_{2}\right), n_{0}=4, n_{1}=n_{2}=5$, has no torsion but is not shellable. Glenn Iba has constructed generalized Prüfer codes to count shellable trees. That is how we know guesses (20) and (21) are wrong where they have not been proved right. There are relations among the number of codes, the number of trees, and the numbers in (20) and (21) which are not yet understood. In general, 
in counting subcomplexes, if anything can happen it will. That is because any $r$-dimensional simplicial complex $L$ is clearly a subcomplex of the $r$-skeleton of the $\# L_{0}-1$-simplex, and the barycentric subdivision of $L$ is a subcomplex of $K\left(N_{0}, \ldots, N_{r}\right)$ when $\# N_{i}=\# L_{i}$.

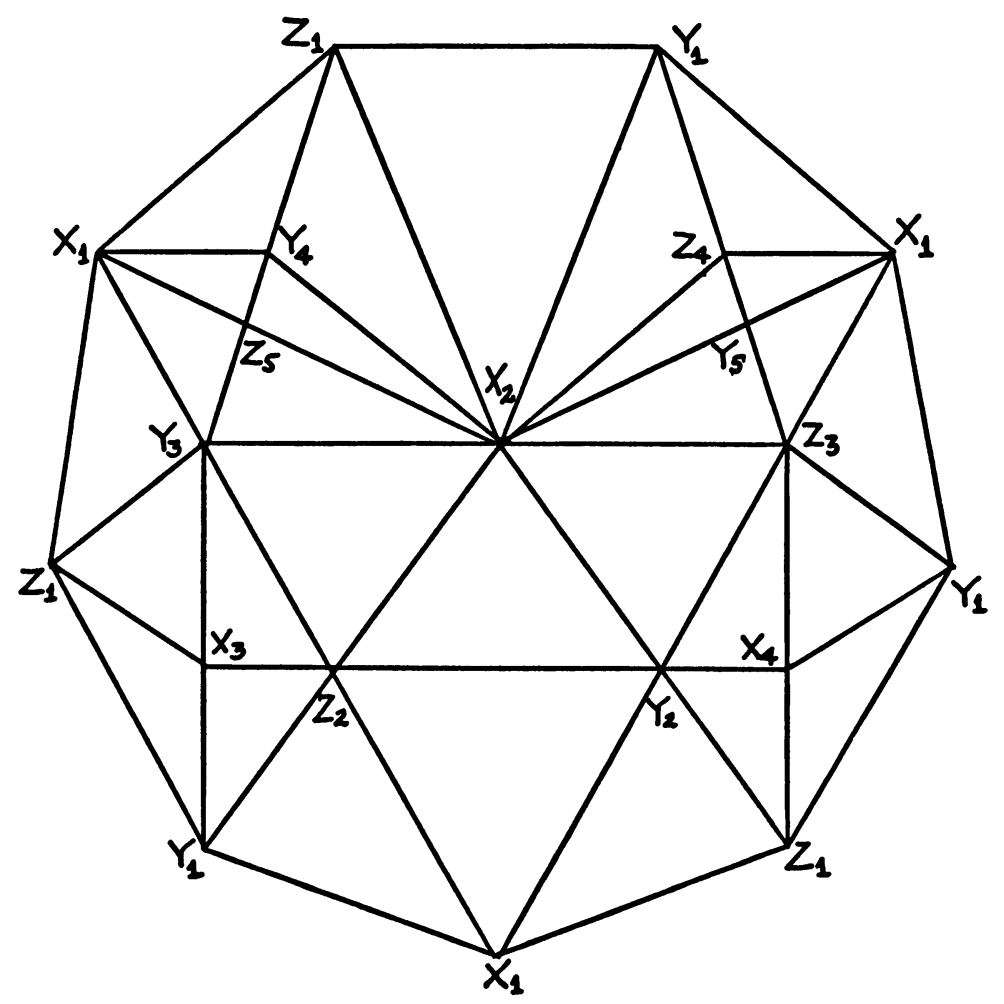

Figure 2

The dunce's cap

5. Face lifting and the $j$-sum problem. In this section we shall construct a cell complex $\mathrm{K}$ analogous to $K\left(N_{0}, \ldots, N_{r}\right)$ with which we can study the $j$-sum problem for $j>1$ as we have already studied the line sum problem. Let $\Gamma^{r}$ be the $r$-cell obtained by truncating each face of the regular $r$-simplex $\Delta^{r}$ in Euclidean $r$-space. Figure 3 shows $\Gamma^{3}$. In general, $\Gamma^{r}$ is dual to the barycentric subdivision of $\Delta^{r}$. If we identify $\Delta^{r}$ with the set $\{0,1, \ldots, r\}$ of its vertices then each proper subset $I$ of $\Delta^{r}$ determines a face of $\Delta^{r}$ of dimension $\# I-1$ and a facet $F_{I}$ of $\Gamma^{r}$. That is why we call $\Gamma^{r}$ a face lifting of $\Delta^{r}$. If we let the hyperplanes which truncate $\Delta^{r}$ to yield $\Gamma^{r}$ recede continuously, we see a continuous deformation of $\Gamma^{r}$ into $\Delta^{r}$ which simultaneously shrinks each $F_{I}$ to $I$. We call that opera- 
tion untruncating. Every permutation $\alpha=\left(\alpha_{0}, \ldots, \alpha_{r}\right)$ of the vertices of $\Delta^{r}$ defines a vertex of $\Gamma^{r}$ : the vertex $\alpha$ is on the facet $F_{I}$ if and only if $I=$ $\left\{\alpha_{0}, \ldots, \alpha_{\# I}\right\}$. Two faces $I$ and $J$ of $\Delta^{r}$ meet if and only if $I \cap J \neq \varnothing$. Two facets $F_{I}$ and $F_{J}$ of $\Gamma^{r}$ meet if and only if $I \subset J$ or $J \subset I$.

Suppose $I \subset \Delta^{r}$. Then $E_{I}=\bigcup_{J C I} F_{J}$ is homeomorphic to an $r-1$-cell on the boundary of $\Gamma^{r}$. When we untruncate, $E_{I}$ shrinks to $I$.

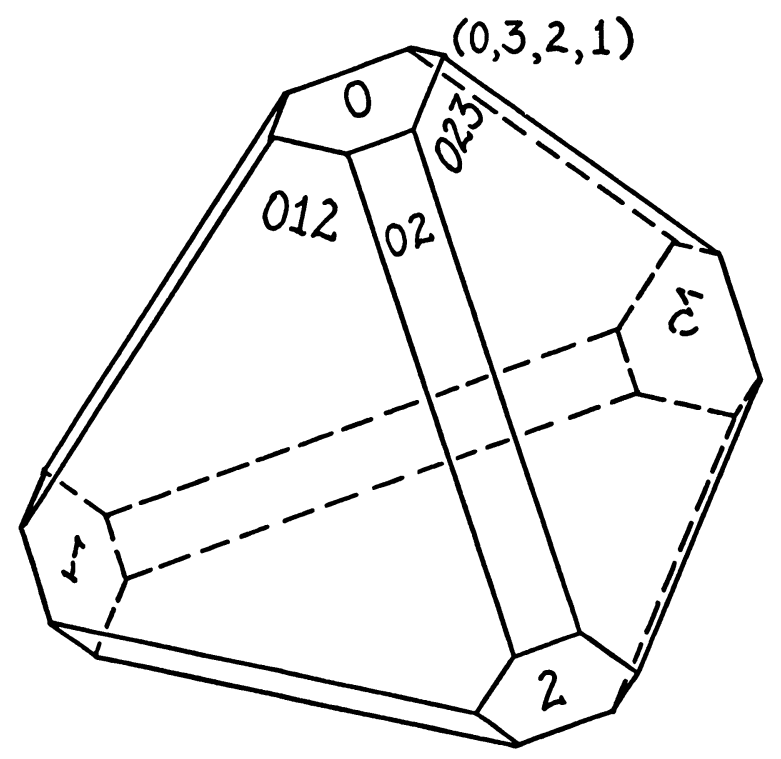

FIGURE 3

$\Gamma^{3}$

Now let $N_{0}, \ldots, N_{r}$ be as before. When we built $K\left(N_{0}, \ldots, N_{r}\right)$ we started with an $r$-simplex for each $\sigma \in K=N_{0} \times \cdots \times N_{r}$. Now we shall start instead with an oriented copy of $\Gamma^{r}$ for each such $\sigma$, and shall call that copy $\bar{\sigma}$. When $\sigma, \sigma^{\prime} \in K$, let $I=\left\{i \mid \pi_{i}(\sigma)=\pi_{i}\left(\sigma^{\prime}\right)\right\}$. Then paste together $\bar{\sigma}$ and $\bar{\sigma}^{\prime}$ by identifying $E_{I}(\sigma)$ with $E_{I}\left(\bar{\sigma}^{\prime}\right)$, preserving orientation. We call the resulting cell complex $\mathbf{K}=\mathbf{K}\left(N_{0}, \ldots, N_{r}\right)$, the face lifting of $K$. One can perform this face lifting operation on any coherently oriented, purely $r$-dimensional simplicial complex. The resulting cell complex is finite, normal, regular, coherently oriented and purely $r$-dimensional. Thus all of $\S 3$ applies to it.

LEMma 29. $\mathrm{K}$ and $\mathrm{K}$ have the same homotopy type, and hence the same homology.

Proof. Simultaneously untruncating each cell $\bar{\sigma}$ of $\mathbf{K}$ establishes the required homotopy equivalence. 
LEMMA 30. $(I, \tau)$ is a facet of $\bar{\sigma} \in K$ if and only if $\pi_{I}(\sigma)=\tau$, or, equivalently, $\sigma$ lies on the subarray $\tau$.

Proof. Just unravel the definitions; compare Lemma 1.

Lemma 30 establishes a natural correspondence between $K_{r-1}$ and $\bigcup_{i<r-1} K_{i}$. The next theorem exploits that correspondence to show that the boundary operator of $\mathbf{K}$ gives more visible information than that of $K$.

THEOREM 31. When the matrix $f$ is regarded as an $r$-chain on $\mathrm{K}$ and $(I, \tau) \in \mathbf{K}_{r-1}$ then

$$
\partial f(\tau)=\sum_{\pi_{I}(\sigma)=\tau} f(\sigma)
$$

the $j$-sum of $f$ on the $j$-subarray $\tau$, where $j=r+1-\# I$.

Proof. Use Lemma 30 and (2) and (3).

When we are interested in the $j$-sums of a matrix we shall want to shrink the elements of $\mathrm{K}_{r-1}$ which correspond to $i$-subarrays for $i<j$. To that end we define shrinking operators $S_{i}$ on the cell $\Gamma^{r}$.

Construction 32. Let $S_{0}$ be the identity and $\Gamma_{(1)}^{r}=\Gamma^{r}$. The operator $S_{1}$ retracts to a point each of the $r+1$ disjoint facets $F_{I}$ of $\Gamma_{(1)}^{r}$ for which $\# I=r$. The resulting $r$-cell $\Gamma_{(2)}^{r}$ has a facet $F_{I}$ for each $I$ for which $\# I \leqslant r-1$. In Figure 4 we have sketched $\Gamma_{(2)}^{3}$. On $\Gamma_{(2)}^{r}$ there are $\left(\begin{array}{c}+1 \\ 2\end{array}\right)$ facets $F_{I}$ for which $\# I=r-1$. Each of these is an $r-1$-cell on whose boundary lie two distinguished points, the remnants of the two facets $F_{J}$ of $\Gamma_{(1)}^{\prime}, J \supset I$, which $S_{1}$ collapsed to points. Those two points are the boundary of a 1 -cell $I^{1}$ essentially orthogonal to the $r-2$-cell $I$ in $F_{I}$. Thus $F_{I}$ in $\Gamma_{(2)}^{r}$ is naturally a suspension of $I$. The shrinking operator $S_{2}$ retracts each $F_{I}$ in $\Gamma_{(2)}^{r}$ for which $\# I=r-1$ to the 1-cell $I^{1}$. The resulting $r$-cell is $\Gamma_{(3)}^{r}$. Figure 4 shows $\Gamma_{(3)}^{3}$.

Inductively, suppose the shrinking operator $S_{j-1}$ has been defined so that its effect on $\Gamma_{(j-1)}^{r}$ is to produce an $r$-cell $\Gamma_{(j)}^{r}$ with the following properties. There is a facet $F_{I}$ of $\Gamma_{(j)}^{r}$ for each $I$ for which $\# I \leqslant r+1-j$. For $\left(\begin{array}{c}r+1 \\ j\end{array}\right)$ of those $I$, $\# I=r+1-j$. Each of those $F_{I}$ is an $r-1$-cell whose boundary contains a distinguished $j-2$-sphere, the remnant of the facets $F_{J}$ of $\Gamma^{r}$ with $J \supset I$, which were shrunk by earlier operators. That $j-2$-sphere is the boundary of a $j-1$-cell $I^{1}$ essentially orthogonal to the $r-j$-cell $I$ in $F_{I}$. Thus $F_{I}$ in $\Gamma_{(j)}^{r}$ is naturally a $j-1$-fold suspension of $I$. The shrinking operator $S_{j}$ retracts each $F_{I}$ with $\# I=r+1-j$ to the $j-1$-cell $I^{\perp}$.

We shall write $\mathbf{K}_{(j)}$ for the cell complex which results when we apply the shrinking operators $S_{1}, \ldots, S_{j-1}$ sequentially to each of the copies $\bar{\sigma}$ of $\Gamma^{r}$ from which $\mathbf{K}$ is built. Do not confuse $\mathbf{K}_{(j)}$ with $\mathbf{K}_{j}$, the $j$-skeleton of $\mathbf{K}$. Next we compute the homology of $\mathbf{K}_{(j)}$. 


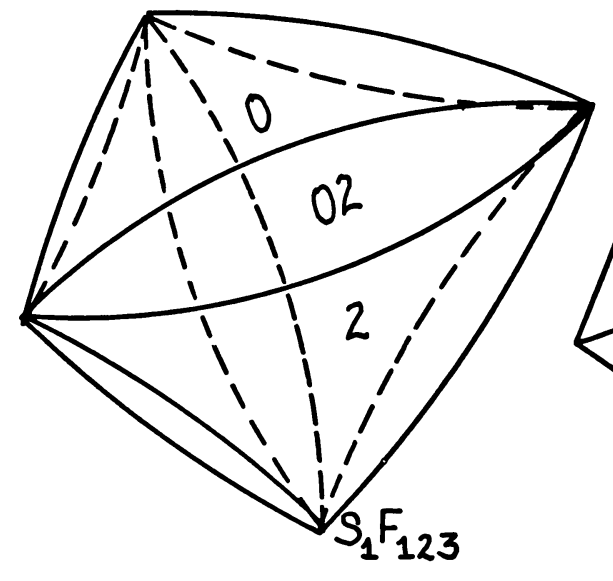

$\Gamma_{(2)}^{3}$

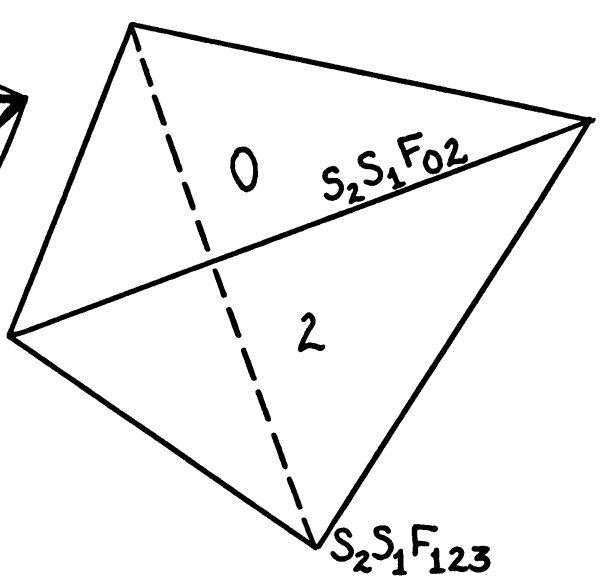

$\Gamma_{(3)}^{3}$

FIGURE 4

THEOREM 33. $H_{d}\left(\mathbf{K}_{(j)}\right)$ is free of rank 0 if $d \neq r$ and of rank

$$
\chi_{(j)}=P_{r+1}-\sum_{q=0}^{r+1-j}(-1)^{q}\left(\begin{array}{c}
j+q-1 \\
q
\end{array}\right) P_{r+1-j-q}
$$

when $d=r$. Here $\chi_{(j)}$ is just $\chi\left(\mathbf{K}_{(j)}\right)$.

Proof. When $j=1$ the theorem says

$$
\chi(\mathrm{K})=P_{r+1}-P_{r}+P_{r-1}-\cdots=\prod_{i=0}^{r}\left(n_{i}-1\right)
$$

which is Theorem 4 . When $j=2$ it says

$$
\chi\left(\mathbf{K}_{(2)}\right)=P_{r+1}-P_{r-1}+2 P_{r-2}-3 P_{r-3}+\cdots
$$

which confirms Corollary 3.4 of [4] in which the dimension of the answer to a plane sum problem when $r=2$ is shown not to exceed $n_{0} n_{1} n_{2}-n_{0}-n_{1}-n_{2}$ +2 . When $j=r+1$ the theorem says

$$
\chi_{(r+1)}=n_{0} n_{1} \cdots n_{r}-1,
$$

which is clearly correct for the dimension of the answer to an $(r+1)$-sum problem: if the sum of all the elements of a matrix is specified then the space of matrices is of codimension 1.

To prove the theorem we need only its correctness for $j=1$, shown above, and the following induction on $j$. Suppose (22) correct for $\chi_{(j)}$. To obtain 
$\mathbf{K}_{(j+1)}$ we apply the shrinking operator $S_{j}$ to each of the r-cells of $\mathbf{K}_{(j)}$. Suppose $I=\left\{i_{0}, \ldots, i_{r-j}\right\}$; there are $\left(\begin{array}{c}r+1 \\ j\end{array}\right)$ such subsets of $\Delta^{r}$. Let

$$
L_{I}=\left\{F_{I}\left(\bar{\sigma}_{(j)}\right) \mid \sigma \in K\right\} .
$$

Then $L_{I}$ is a subcomplex of the $r-1$-skeleton of $\mathrm{K}_{(j)}$. Construction 32 shows $L_{I}$ is just the $j$-fold suspension of $K\left(N_{i_{0}}, \ldots, N_{i_{r-j}}\right)$. Then Theorem 4 implies $H_{d}\left(L_{I}\right)$ is free of rank $\left(n_{i_{0}}-1\right) \cdots\left(n_{i_{r-j}}-1\right)$ if $d=(r-j)+(j-1)$ $=r-1$ and is $\{0\}$ otherwise. When we apply $S_{j}$ to $\mathbf{K}_{(j)}, L_{I}$ retracts to a single $j-1$-cell $I^{\perp}$, which is contractible to a point. Thus

$$
H_{d}\left(\mathbf{K}_{(j)}, S_{j} L_{I}\right) \approx H_{d}\left(\mathbf{K}_{(j)}, L_{I}\right) .
$$

We can then infer from the exact relative homology sequence

$$
\cdots \rightarrow H_{d}\left(S_{j} L_{I}\right) \rightarrow H_{d}\left(\mathbf{K}_{(j)}\right) \rightarrow H_{d}\left(\mathbf{K}_{(j)}, S_{j} L_{I}\right) \rightarrow H_{d-1}\left(S_{j} L_{I}\right) \rightarrow \cdots
$$

that

$$
H_{d}\left(\mathbf{K}_{(j)}, S_{j} L_{I}\right) \approx H_{d}\left(\mathbf{K}_{(j)}\right) \times H_{d-1}\left(S_{j} L_{I}\right),
$$

so that $H_{d}\left(\mathbf{K}_{(j)}, S_{j} L_{I}\right)$ is free of rank $\chi_{(j)}+\left(n_{i_{0}}-1\right) \cdots\left(n_{i_{r-j}}-1\right)$ if $d=r$ and is $\{0\}$ otherwise.

Since $S_{j}$ shrinks each $F_{I}$ to $I^{\perp}$ independently of the others, it follows that $H_{d}\left(\mathrm{~K}_{(j+1)}\right)$ is $\{0\}$ if $d<r$ such that $H_{r}\left(\mathrm{~K}_{(j+1)}\right)$ is free of rank

$$
\begin{aligned}
\chi_{(j+1)} & =\chi_{(j)}+\sum_{\# I=r+1-j} \prod_{i \in I}\left(n_{i}-1\right) \\
& =\chi_{(j)}+\sum_{q=0}^{r+1-j}(-1)^{q}\left(\begin{array}{c}
j+q \\
q
\end{array}\right) P_{r+1-j-q} \\
& =P_{r+1}-\sum_{q=0}^{r+1-j}(-1)^{q}\left[\left(\begin{array}{c}
j+q-1 \\
q
\end{array}\right)-\left(\begin{array}{c}
j+q \\
j
\end{array}\right)\right] P_{r+1-j-q} \\
& =P_{r+1}-\sum_{q=0}^{r+1-j}(-1)^{q+1}\left(\begin{array}{c}
j+q-1 \\
q-1
\end{array}\right) P_{r+1-j-q} .
\end{aligned}
$$

When we replace $q$ by $q+1$ in the last member of (24), remembering that $\left(\begin{array}{c}m \\ 0\end{array}\right)$ $=1$ and $\left(\begin{array}{c}m \\ -1\end{array}\right)=0$, we get the right member of $(22)$ for $\mathrm{K}_{(j+1)}$.

Our last task is to show that the complex $\mathbf{K}_{(j)}$, to which all of $\S 3$ applies, is the right one for studying the $j$-sum problem.

LEMMA 34. If the j-sums of a matrix are known then values for all the $i$-sums, $i \geqslant j$, can be computed.

Proof. It suffices to show how to compute the $j+1$-sums. Let $(I, \tau)$ be a $j+1$-subarray. Pick any $i \notin I$. Then 


$$
\sum_{\pi_{I}(\sigma)=\tau} f(\sigma)=\sum_{v \in N_{i}} \sum_{\pi_{i}(\sigma)=v ; \pi_{I}(\sigma)=\tau} f(\sigma)
$$

so each $j+1$-sum is a sum of $j$-sums.

THEOREM 35. Let $g \in C_{r j}(K)$. Then $g$ can be thought of as a function on $K_{r-j} \subset\left(\mathrm{K}_{(j)}\right)_{r-1}$. When so regarded, $g$ extends to an $r-1-c y c l e$ on $\mathbf{K}_{(j)}$ if and only if there is a matrix $f$ whose $j$-sums are given by $g$. In that case the extension is unique, and $\partial^{-1} g$ is the set of all matrices with $j$-sums $g$; it has dimension $\chi_{(j)}$. Thus when $g \geqslant 0, \chi_{(j)}$ is the maximum possible dimension for $M(g)$.

Proof. If $g$ gives the $j$-sums of $f$ then Theorem 31 shows the $r-1$-cycle $\partial f$ on $\left(K_{(j)}\right)_{r-1}$ extends $g$. Conversely, if $g$ extends to a cycle, which we shall still call $g$, then since $H_{r_{-1}}\left(\mathbf{K}_{(j)}\right)=\{0\}, \partial g=0$ implies $\partial^{-1} g$ has dimension $\chi_{(j)}=\operatorname{rank} H_{r}\left(\mathbf{K}_{(j)}\right)$. Then for any $f \in \partial^{-1} g, \partial f=g$ so the extension of $g$ is unique. In fact, Lemma 34 tells how to extend $g$; the condition $\partial g=0$ says precisely that the computation in that lemma is independent of the choice of $i \notin I$.

ACKNOWLEDGment. "That's wonderful. But now you have to learn more topology." So ended each valuable, provocative talk with Victor Guillemin. He thought I would need spectral sequences for §5; perhaps I should have taken his advice.

\section{REFERENCES}

1. E. D. Bolker, Transportation polytopes, J. Combinatorial Theory, 13(1972), 251262.

2. Counting trees in simplicial complexes, Notices Amer. Math. Soc. 21 (1974), A-32. Abstract \#711-05-12.

3. H. Crapo and G. C. Rota, On the foundations of combinatorial theory: Combinatorial geometries, Preliminary edition, M.I.T. Press, Cambridge, Mass., 1970. MR 45 \#74.

4. W. B. Jurkat and H. J. Ryser, Extremal configurations and decomposition theorems, J. Algebra 8 (1968), 194-222. MR 36 \#3667.

5. V. Klee and C. Witzgall, Facets and vertices of transportation polytopes, Lectures in Appl. Math., vol. 11, Amer. Math. Soc., Providence, R. I., 1968, pp. 257-282. MR 38 \#4134.

6. A. T. Lundell and S. Weingram, The topology of CW complexes, Van Nostrand Reinhold, New York, 1969.

7. E. H. Spanier, Algebraic topology, McGraw-Hill, New York, 1966. MR 35 \#1007.

8. E. C. Zeeman, On the dunce hat, Topology 2 (1963), 341-358. MR 27 \#6275.

9. H. Bruggesser and P. Mani, Shellable decompositions of cells and spheres, Math. Scand. 29 (1971), 197-205. MR 48 \#7286.

DEPARTMENT OF MATHEMATICS, UNIVERSITY OF MASSACHUSETTS, BOSTON, MASSACHUSETTS 02125 